\title{
Emerging Therapeutic Options for COVID-19
}

\author{
Sheetal Rameshlal Khubchandani ${ }^{1}$, Trupti Madhav Dahane ${ }^{2}$ \\ 1,2 Department of Prosthodontics, Crown and Bridge, Sharad Pawar Dental College, \\ DMIMS (DU), Sawangi, Wardha, Maharashtra, India.
}

\section{ABSTRACT}

\section{BACKGROUND}

COVID-19, the "pandemic", "public health crisis" etc., are the news headlines every morning which is threatening the people and developing a feeling of fear and anxiety amongst the people in almost all the countries across the globe. Scientists, doctors and all other health care professionals are making continuous and tremendous efforts in searching and developing different ways to control the outbreak of the pandemic viral disease. Also, development of new drugs, testing efficacy, and clinical trials of the old available drugs and efforts for development of vaccination for COVID-19 to prevent its occurrence in the near future is still ongoing. But unfortunately, there exists no single particular approved / recognized treatment or drug for the COVID19 patients yet. This review article is aimed at enlightening most of the preventive measures and treatment options that are available and described in the literature.

\section{KEY WORDS}

Covid-19, SARS, Prevention, Treatment.
Corresponding Author: Dr. Sheetal Rameshlal Khubchandani. Near S T Depot, Ramnagar,

Wardha-442001, Maharashtra, India. E-mail:dr.srkhubchandani@gmail.com

DOI: $10.14260 /$ jemds $/ 2020 / 677$

How to Cite This Article:

Khubchandani SR, Dahane TM. Emerging therapeutic options for COVID-19. J Evolution Med Dent Sci 2020;9(41):30823085, DOI: 10.14260/jemds/2020/677

Submission 30-04-2020,

Peer Review 02-09-2020,

Acceptance 09-09-2020,

Published 12-10-2020.

Copyright (C) 2020 Dr. Sheetal Rameshlal Khubchandani et al. This is an open access article distributed under Creative Commons Attribution License [Attribution 4.0 International (CC BY 4.0)] 


\section{BACKGROUND}

Corona Virus Disease - 2019 or COVID-19 which was earlier known as 2019 novel corona virus (2019 - nCoV) or severe acute respiratory syndrome corona virus 2 (SARS - CoV-2) originated in animals such as bats and spread throughout the world. First evidence of such disease is from Wuhan city, Hubei Province, China in the month of December 2019. Emergence of this virus from China to other countries of the world is considered as life-threatening public health crisis worldwide and later was announced as pandemic by WHO (World Health organization) ${ }^{1}$

The clinical characteristics of COVID-19 are unpredictable that ranges from no symptoms to acute respiratory distress syndrome (ARDS) and multi organ dysfunction. Fever, cough, sore throat, headache, fatigue, muscle pain and difficulty in breathing are the commonest clinical features. Conjunctivitis seen in some patients. Thus, it is difficult to distinguish it from other respiratory infections. In a division of patients, in the first week or its end, the disease can progress to pneumonia or respiratory failure and eventually death. It affects elderly population more as compared to young. This is because aging is associated with increased risk of functional dependence leading to frequent hospitalization and increased mortality. This in turn leads to poor quality of life and thus there is need for long term and higher healthcare facilities. ${ }^{2,3}$

Different treatment options starting from symptomatic treatment to the definitive one and from simple pharmacological agents or supportive therapies to the invasive therapies are mentioned in the literature and also tried in the positive patients as well as suspects of COVID-19. In-vitro studies are also carried out for development of new drugs and vaccines. This article focuses on compilation of all such available treatment options and discussing their efficacy and effectiveness in the treatment of COVID-19. Also, we are not attempting to recommend any of the treatment as none is approved by the WHO yet.

\section{Prevention}

Prevention is better than cure. As rightly quoted, the first step in the treatment of COVID-19 is its prevention and control of the spread. Transmission of the virus from one human to another can be prevented by certain simple tools of prevention. It includes three important aspects; a) Isolation b) Quarantine c) Social Distancing and Community containment. ${ }^{4}$ Adequate isolation play crucial role in prevention of disease by restricting the transmission of the virus from one person to another. ${ }^{1}$

\footnotetext{
Adequate Isolation

Isolation can be described as the separation of the infected persons from the non-infected ones or healthy individuals or non-infected but patients with debilitating diseases who are prone to any opportunistic infection. Isolation rooms with negative pressure in the hospital set ups are helpful in this purpose. ${ }^{4}$ As the incubation period of the disease is longer isolation is must to reduce the shedding of the virus during the incubation period. The mean incubation period was found as 5.2 days to 12.5 days at different confidence intervals in a study by Qun Li. ${ }^{5}$
}

\section{Quarantine}

It is an important tool in controlling of any communicable disease since olden times. Quarantine implies interruption in the social contact. People are restricted at their present place till the situation gets under control. This practice was done in Italy in way back in $14^{\text {th }}$ century when ships arriving at Venice port with plaque-infected passengers the ships were anchored at ports. ${ }^{6}$ Also, in the year 2003, during the SARS epidemic in China quarantine was implemented and it was a successful preventive measure for control of SARS disease. ${ }^{7}$ As the clinical features of COVID-19 are similar to SARS, quarantine is considered as effective preventive measure here also.

\section{Social Distancing and Community Containment}

Reduction in the interactions between people is termed as social distancing. It helps in prevention of transmission of virus in people who are yet to be diagnosed or asymptomatic. Also due to failure of identification of such patients; they are not isolated but carry a potential risk of transmission. Social distancing is important tool in such cases. ${ }^{4}$ These measures are considered important where disease is known to have a community spread. But it is not sufficient in controlling the disease, community containment should be practiced which means ensuring only vital supplies and restricting a wide population such as a region, place or city. ${ }^{8}$

\section{TREATMENT OPTIONS FOR COVID-19}

\section{Symptomatic or Supportive Therapy}

As definitive treatment is yet to be developed and approved, the first line therapy for the COVID-19 positive patients is the symptomatic relief of the symptoms. Supportive therapy is important for boosting the immunity. It includes proper nutrition and hydration of the body. Screening of the patients with symptoms but negative for viral testing should be considered.

\section{Respiratory Support}

Under respiratory support comes oxygenation. According to the "Diagnosis and Treatment of Pneumonia caused by COVID19" (updated to version 6) issued by National Health Commission of the People's Republic of China currently due to lack of antiviral therapy, supportive and respiratory treatment is focused. ${ }^{2}$ Decreased ventilation causes increase in $\mathrm{pCO}_{2}$ and decrease in $\mathrm{pO}_{2}$ levels. During apnoea stores of oxygen falls rapidly. So, oxygenation helps in maintaining the balance. ${ }^{9,10}$ Almost all positive patients of COVID-19 responded well to the oxygen therapy as they mainly suffer from hypoxia and pneumonia like symptoms. For the patients with refractory hypoxemia, WHO recommended ECMO therapy that is Extracorporeal Membrane Oxygenation. ${ }^{11}$

\section{Pharmacological Agents}

No particular pharmacological agents or drugs are currently approved for Corona Virus Disease-2019 (COVID-19). Although some authors have been tried, recommended and done some clinical trials using the available drugs. These drugs are described in the literature by many authors according to 
their effectiveness in treatment of COVID-19 cases. Some of them are described as follows:

\section{Anti-Viral Agents}

As we know no drug is yet approved for COVID-19. Some of the anti-viral agents that were previously used and successfully cured diseases such as SARS (Severe Acute Respiratory Syndrome) and MERS (Middle East Respiratory Syndrome) , on the basis of experience of these drugs in SARS and MERS they are also tried in COVID-19 cases and the results were variable. Drugs which were used includes the protease inhibitors such as ribavirin and lopinavir-ritonavir. Studies by Chen $\mathrm{N}$ et al. have shown that ribavirin alone was less effective. Outcomes were enhanced when ribavirin was used along with lopinavir-ritonavir combination. ${ }^{12}$

In other studies, antiviral agents such as neuraminidase inhibitors-oseltamivir, ganciclovir, lopinavir-ritonavir were used in combination therapy and were given to children. Results of the study by Xu XW showed that all the children recovered and there was no need of intensive care in such children who were on this antiviral therapy. ${ }^{13}$ Such antivirals either existing or new antivirals can respond rapidly but one should consider the neurological side effects due to them. In a study by Balwani $\mathrm{M}$ et al. they used 2 new antivirals for treatment of HCV (Hepatitis C Virus) infection which were sofosbuvir and daclatasvir but they stopped it later because data was not available about the neurological side effects. ${ }^{14}$ Further studies and clinical trials are required to recommend a particular anti-viral drug.

\section{Anti-Malarial Agents}

Chloroquine and hydroxychloroquine are the known antimalarial drugs and are commonly used for the treatment of malaria from many years till date. ${ }^{15}$ Studies and clinical trials showed that this drugs are effective in the treatment of SARS as well as COVID-19. Various mechanisms of action were described for the working mechanism of the chloroquine in viral diseases other than malaria. Mechanism of action includes inhibition of $\mathrm{pH}$-dependent phases in the process of replication of the viral DNAs, immunomodulatory effects such as suppression of the factors like TNF-alpha and Interleukein $6 .^{2}$ Combination of certain drugs with chloroquine have also shown improved results than using chloroquine alone. For example, when chloroquine was used along with azithromycin (a drug used for severe respiratory diseases and upper respiratory tract infections) in an open-label non-randomized clinical trial, results showed that this is a synergistic combination and is effective against COVID-19. Also azithromycin is known to be effective against EBOLA virus as well.16 Other authors also mentioned use of systemic corticosteroid treatment such as methylprednisolone ${ }^{17}$, antifungal agents and monoclonal antibodies therapy ${ }^{18}$ for the treatment of COVID-19. Success rates were variable and no promising benefits were mentioned.

\section{Convalescent Plasma Therapy}

Kai Duan et al. in their study checked the viability of convalescent plasma (CP) to treat COVID positive patients. The results were obtained from 10 cases and showed that one dose of CP - $200 \mathrm{ml}$ was well tolerated by the patients. Also, it could significantly enhance the neutralizing antibodies which in turn leads to disappearance of viremia in 7 days. Clinical symptoms and para-clinical criteria rapidly improved within 3 days. These results indicate that $\mathrm{CP}$ can serve as a promising rescue option for COVID-19 but other studies and recent updates also stated that there are complications. So it is still a controversy. The optimal dose, time, clinical benefit of CP therapy needs further long-term investigation in well controlled trials. ${ }^{19}$ Further clinical trials and studies are required to come to a concrete conclusion.

\section{CONCLUSIONS}

COVID-19 outbreak has challenged social, economic, medical as well as public health sectors of almost all the countries of the world. Rather than developing fear and anxiety, it is the duty of each and every individual to prevent and control the spread at individual level. As a health care professional, responsibility is little more on us. Future impact of the condition is still questionable. Therefore, apart from complaining about the difficulties that we are facing due to social distancing and lockdown, efforts should be made to prevent the further spread and extension of the pandemic. Further interventional studies and clinical trials for the approved drugs for COVID-19 are required. Early development of the vaccination will also help in reducing anxiety amongst people.

Financial or Other Competing Interests: None.

\section{REFERENCES}

[1] Singhal T. A review of coronavirus disease- 2019 (COVID19). Indian J Pediatr 2020;87(4):281-6.

[2] Guo YR, Cao QD, Hong ZS, et al. The origin, transmission and clinical therapies on coronavirus disease 2019 (COVID-19) outbreak - an update on the status. Mil Med Res 2020;7(1):11.

[3] Agrawal A, Kumar S, Bhagwati J. Correlation of platelet indices with clinical profile in elderly patients: a study in rural teaching hospital. Ann of Med Healt Sci Res 2018;8(3):163-69.

[4] Wilder-Smith A, Freedman DO. Isolation, quarantine, social distancing and community containment: pivotal role for old-style public health measures in the novel coronavirus (2019-nCoV) outbreak. J Travel Med 2020;27(2):taaa020.

[5] Li Q, Guan X, Wu P, et al. Early transmission dynamics in Wuhan, China, of novel coronavirus- infected pneumonia. N Engl J Med 2020;382(13):1199-207.

[6] Cetron M, Simone P. Battling 21st-century scourges with a $14^{\text {th }}$ century toolbox. Emerg Infect Dis 2004;10(11):2053-4.

[7] Goh KT, Cutter J, Heng BH, et al. Epidemiology and control of SARS in Singapore. Ann Acad Med Singap 2006;35(5):301-16. 
[8] Appendix D1: interventions for community containment. https://www.cdc.gov/sars/guidance/dquarantine/app1.htm.

[9] Parag P, Acharya S, Shukla S. Cheyne stokes breathing in a case of tubercular meningitis with hydrocephalus. J Clin Diagn Res 2019;13(5):0J01-2.

[10] Prasad TM, Ninave SS. Prospective comparison of pressor and airway responses to iv esmolol and iv dexmedetomidine during emergence from general anaesthesia and extubation. J Krishna Institute Med Sci Univers 2017;6(1)49-56.

[11] WHO. Clinical management of severe acute respiratory infection when novel coronavirus (nCoV) infection is suspected. https://www.who.int/publicationsdetail/ clinical-management-of-severe-acute-respiratoryinfection-when-novelcoronavirus-(ncov)-infection-issuspected.

[12] Chen N, Zhou M, Dong X, et al. Epidemiological and clinical characteristics of 99 cases of 2019 novel coronavirus pneumonia in Wuhan, China: a descriptive study. Lancet 2020;395(10223):507-13.

[13] Xu XW, Wu XX, Jiang XG, et al. Clinical findings in a group of patients infected with the 2019 novel coronavirus (SARS-Cov-2) outside of Wuhan, China: retrospective case series. BMJ 2020;368:m606.
[14] Balwani M, Bawankule C, Ramteke V, et al. Hepatitis C virus, directly acting antivirals and guillain-barre syndrome. Saudi J Kidney Dis Transpl 2018;29(5):12379.

[15] Aguiar ACC, Murce E, Cortopassi WA, et al. Chloroquine analogs as antimalarial candidates with potent in vitro and in vivo activity. Int J Parasitol Drugs Drug Resist 2018;8(3):459-64.

[16] Gautret P, Lagier JC, Parola P, et al. Hydroxychloroquine and azithromycin as a treatment of COVID-19: results of an open-label non-randomized clinical trial. Int J Antimicrob Agents 2020;56(1):105949.

[17] Wang D, Hu B, Hu C, et al. Clinical characteristics of 138 hospitalized patients with 2019 novel coronavirusinfected pneumonia in Wuhan, China. JAMA 2020;23(11):1061-9.

[18] Shanmugaraj B, Siriwattananon K, Wangkanont K, et al. Perspectives on monoclonal antibody therapy as potential therapeutic intervention for coronavirus disease- 19 (COVID-19). Asian Pac J Allergy Immunol 2020;38(1):10-8.

[19] Duan K, Liu B, Li C, et al. Effectivenss of convalescent plasma therapy in severe COVID-19 patients. Proc Natl Acad Sci U S A 2020;117(17):9490-6. 